\section{Zwischen Skylla und Charybdis}

\author{
Ein Gespräch mit Dr. Hans Heinrich Brunner zu aktuellen Themen \\ der Gesundheits- und ärztlichen Berufspolitik
}

Wer den FMH-Präsidenten von "TARMED" her kennt, wird vielleicht überrascht sein, ihn hier von einer ganz anderen Seite zu erleben: als Arzt und Politiker auf der Höhe seiner Zeit.

\section{SÄZ: Wenn ich die Schweizer Gesundheitspolitik betrachte, so kann ich kaum ernsthafte Bemühungen erkennen, die sich dem Gemeinwohl verpflichtet fühlen. Ich sehe überall Kleinkrämer, die sich um reine Partikularinteressen scheren - sehe ich das falsch?}

Dr. H. H. Brunner: Diese Sichtweise gibt ein grosses und weitverbreitetes Missbehagen wieder, das ich bei Patienten, Politikern und Medienleuten feststelle. Der Eindruck der Kleinkrämerei kann entstehen, weil die Partikularinteressen im Gesundheitswesen in den letzten Jahren ausgesprochen stark zum Tragen gekommen sind. Im Gesundheitssystem wird einer langjährigen schweizerischen Tradition folgend starker Minoritätenschutz betrieben, der zur Vertretung von Partikularinteressen einlädt. Die gleiche Art der Partikularinteressenvertretungen stellen wir in Teilen der Wirtschaft fest bis hin zum jüngsten Beispiel des Swissair-Crash.

Kaspar Villiger hat in einem Rückblick auf das letzte Jahr gesagt, mit der Rettung der Swissair sei auch ein Gleichgewicht zwischen Wirtschaft und Staat wieder hergestellt worden. Muss es auch im Gesundheitswesen zu einem Grounding kommen, damit es zwischen Partikularinteressen und Service Public wieder einen Ausgleich gibt?

Ich weiss nicht, ob diese Aussage bezüglich der Swissair und dem Verhältnis der Wirtschaftsinteressen zum Staat à la longue richtig ist. Ich zweifle etwas daran. Ob das, wenn es denn so wäre, auf das Gesundheitswesen übertragbar wäre, wäre noch eine weitere Frage. Ich denke nicht, dass es zu einer Rückverschiebung kommt, weg von den staatlichen Interessen hin zu irgendwelchen Partikular- oder Privatinteressen. Ich sehe aber anderseits auch nicht die geringsten Zeichen am Horizont, die sich mit Medizin als Service Public oder als Public Health beschäftigen würden. Für den Grossteil der Leute aus der Politik ist das kein Thema, wahrscheinlich weil es bis jetzt eine Selbstverständlichkeit war. Die wissen gar nicht, was hier auf dem Spiel steht, weil sie von Kindsbeinen an diesen Service Public zum Nulltarif gewöhnt sind.
Kommt hinzu, dass alle nur ihre Parteifahnen vor sich her tragen ...

Parteifahnen mag sein. Es ist aber auch eine grundsätzliche Entwicklung einer zunehmend arbeitsteiligen Welt, dass sich die Leute für immer kleinere Sektoren zuständig fühlen und von einem bestimmten Punkt an sagen, dass andere Leute Entscheide treffen müssen. Das Problem ist einfach, dass diese «anderen Leute" und insbesondere auch die dazu notwendigen institutionellen Bedingungen in der Schweiz nicht existieren. Die Grundidee ist ja immer noch, dass im Ausgleich aller Partikularinteressen irgendwie ein gescheites Gesamtes, das im Falle den Service Public einschliessen müsste, entstehen würde. Das ist in meinen Augen eine absolut verwegene Hypothese, die vielleicht aufgrund gewisser historischer Gegebenheiten vor einigen Jahrzehnten richtig war, die aber weiter als Selbstverständlichkeit zu nehmen schlichter Unsinn und auch nicht durch Beispiele belegbar wäre.

Dann bleibt uns nur noch übrig, zu resignieren und sich von diesen verschiedenen Kräften treiben zu lassen.

Ich gehe davon aus, dass das Gesundheitswesen durch diejenigen Kräfte, die es jetzt bestimmen, nicht reformierbar ist. Es gibt nur zwei Wege: Entweder kommt es zu einer grösseren Katastrophe - es könnte sein, dass aus irgendwelchen Gründen das Gesundheitswesen wirklich nicht mehr finanzierbar wäre und dann käme es zu dramatischen Veränderungen -, oder den Patienten wird klar, was hier auf dem Spiel steht. Ich denke, dass die Patienten wesentlich aufmerksamer, sensibler und kritischer geworden sind, wegen den Prämienerhöhungen aber auch wegen anderer Vorfälle im Bereich der Medizin. Es ist denkbar, dass dieses Kollektiv, wie es das in der Schweiz immer wieder getan hat, mit einer Initiative für Ordnung sorgt. Aber dass das Parlament, dass die Exekutiven in Bund und Kantonen, dass die sogenannten Partner - nachgerade ein zynischer Begriff - miteinander etwas Segenreiches und Gutes, praktisch gegen Willen der Partikularinteressen, produzieren, ist eine metaphysisch-spekulativ zu nennende Hypothese.

Sehen Sie Ansätze, wie sich die Patienteninteressen gruppieren könnten? Welche Rolle könnten dabei Patientenorganisationen spielen?

Ich glaube nicht, dass die Patientenorganisationen mit ihren gegenwärtigen materiellen und personellen Ressourcen in der Lage sind, eine wichtige Rolle zu spielen, denn sonst hätten sie dies schon lange tun müssen. Ich kann mir vorstellen, dass plötzlich politische Kräfte da sind, die in einer bestimmten Situation beispielsweise eine Initiative einreichen. Das kann sich auch durch einen Zusammenschluss einzelner Flügel verschiedener Parteien ergeben. Es hat schon viele Beispiele dafür gegeben, und das schweizerische System bietet diese Besonderheit, dass das Volk wirklich direkt eingreifen kann. Ich gehe davon 


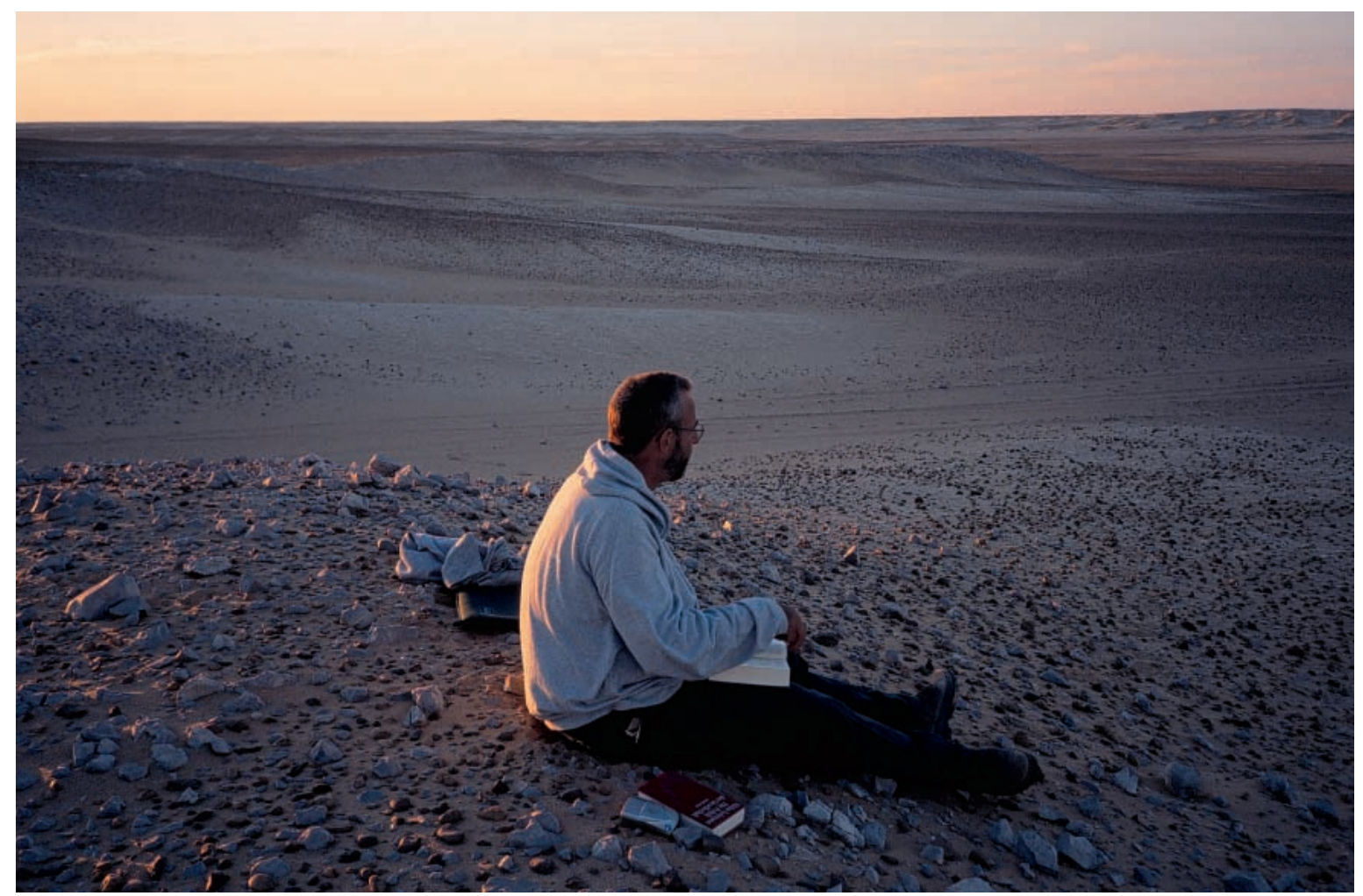

aus, dass das dann geschieht, wenn die Leute genau diese Service-Public-Aufgaben für nicht mehr gegeben oder für nicht mehr realisierbar halten. Dies könnte der Fall sein, wenn die Prämien weiterhin ansteigen wie bisher - auch wenn die Prämien die Kostensteigerung in der Medizin höchst unvollständig widerspiegeln. Auch wenn die Notfallversorgung, die ein zentrales Anliegen des Bürgers darstellt, gefährdet würde, könnte es zu weitreichenden Entwicklungen kommen, wie beispielweise zu einer Initiative.

Wie nahe sind wir an einem solchen Zustand?

Man muss hier zwischen objektiver und subjektiver Ebene unterscheiden. Objektiv müsste ich sagen, dass eigentlich schon längst etwas hätte geschehen müssen. Subjektiv sind sich Bürger und Politiker gar nicht bewusst, in welchem Ausmass das Gesundheitssystems schon Schaden gelitten hat und dass es weiter Schaden leiden wird.

Was braucht es, dass dieses Bewusstsein zustande kommt?

Ich stelle fest, dass in den gegenwärtigen Auseinandersetzungen um die KVG-Revision sehr viele Leute anfangen, sich Gedanken zu machen, und daraus könnte einiges resultieren. Endlich eine öffentliche Diskussion zu führen wird für mich der nächste Markstein sein. Die parlamentarische Kompromisspolitik in der Schweiz täuscht über den wahren Ernst der Sachlage hinweg und verhindert, dass Probleme grundsätzlich erkannt und angegangen werden.
Kennen Sie auch nur einen Politiker, der es wagen würde, zum gegenwärtigen Zeitpunkt das Wort "Rationierung" in den Mund zu nehmen?

Die denken lieber nicht einmal daran ... Nein, ich kenne keinen.

Kennen Sie wenigstens Politiker, welche privat darüber nachdenken?

Ja, aber immer im Sinn des "Gott sei bei uns", also die sagen, Rationierung sei überhaupt das Schlimmste, das passieren könnte, man müsse nur irgendein geniales Rezept finden, um Rationierung zu vermeiden.

\section{Zum Beispiel durch Rationalisierung ...}

Die Leute haben nicht die Andeutung einer Vorstellung, was Rationalisierung im Verhältnis zur Rationierung sein sollte. Es werden immer diese alten blöden Geschichten rumgeboten von den Labor- und Röntgenuntersuchungen, die zweimal gemacht wurden. Als ob es das Problem lösen würde, wenn man solche Dinge zum Verschwinden bringen würde. Mit anderen Worten, ich sehe niemanden in der Schweiz, in den politisch verantwortlichen Kreisen, der dieses Problem wirklich ernst nimmt und sich bemüht, es anzupacken. Es werden immer nur Schönrednerei, Schönfärberei, Betulichkeit und ethischer Schabernack darüber ausgebreitet, aber in der Sache hilft das alles nichts. 
Auf der anderen Seite gibt es die ernstzunehmende Behauptung, Rationierung sei gar nicht nötig, Medizin sei ja schliesslich ein blühender Industriezweig, den man nicht bremsen solle. Liegt denn die Lösung nicht darin, dass wir uns ganz dem medizinischen Kommerz hingeben, in der Hoffnung, dass dann genügend Angebote für alle produziert werden, und erst noch zu einem billigeren Preis? Wir müssen hier wieder auf den Begriff des Service Public zurückkommen. Dieser Sektor ist nach den reinen Gesetzen des Marktes nicht finanzierbar. Wenn es nach diesen Regeln ginge, müssten vor allem sehr teure, aufwändige und preisstarke Produkte hergestellt werden. Alle, die am Markt teilnehmen, wollen möglichst teuer verkaufen. Das ist eigentlich seit Tausenden von Jahren so und weist darauf hin, dass der Markt im Gesundheitswesen nicht ernsthaft Kosten sparen kann. Kommt hinzu, dass gerade im Service Public erbrachte Leistungen nicht dazu bestimmt sind, einen Gewinn abzuwerfen, und damit weder für die pharmazeutische Industrie noch für die medizinische Gebrauchsmittelindustrie und häufig nicht einmal für die Leistungserbringer selbst interessant sind. Markt und Service Public stehen, entgegen allen Schönrednereien, in einem Widerspruch zueinander. Der KVG-Bereich, ein obligatorischer Bereich, der sehr nahe an den Staat herangezogen wurde, muss anders als über Marktkräfte finanziert werden. Daneben gibt es einen Bereich, und das will ich hier ganz deutlich sagen, in welchem Dinge angeboten werden, über die man diskutieren kann, ob sie überhaupt noch zur Medizin gehören - ich denke an alle diese für mich inhaltleeren Floskeln, angefangen bei der Salutogenese bis zu den Wellness-Angeboten. In diesem Bereich kann der Markt herrschen; wenn jemand damit viel Geld verdienen will und erst noch Leute findet, die ihm das Zeug bezahlen, dann soll er das doch machen. Ich halte das keinesfalls für unethisch, solange nicht bewusst Schädigungen oder Nachteile des Kunden, sprich Patienten, in Kauf genommen werden. Der gesamte Bereich der Alternativmedizin ist ein Riesenmarkt, der immer im Schatten der Aufmerksamkeit des Publikums funktioniert hat und auch heute noch so funktioniert.

Sie kommen mir vor wie ein Odysseus, der das Schiff FMH zwischen Skylla - Staat - und Charybdis - Markt - navigieren muss. Aber wohin geht der Kurs?

Ich gehe davon aus, dass wir uns wieder darauf besinnen müssen, was die Patienten von einem Arzt erwarten. Sie erwarten in aller erster Linie, dass sie zu der Person namens Arzt oder Ärztin hingehen können, wenn es ihnen schlecht geht; dass diese Person sich das Problem anhört und die sachliche Kompetenz hat, es zu lösen oder zu sagen, ich kann das nicht, aber ich kenne jemanden, der weiterhelfen kann; dass diese Person dem Patienten in Not zur Seite steht. Das ist seit Bestehen des Codex Hippocraticum der Kern der Medizin, und wir müssen wieder zu dieser Idee zurückfinden und uns nicht zu Gesundheitsmanagern oder Wellnessaposteln missbrauchen lassen.
Warum gelingt es uns Ärzten nicht, diese an sich sehr einfache, klare und überdies noch sehr schöne Botschaft verständlich zu kommunizieren?

Weil wir uns gar nicht darum bemüht haben, weil wir uns viel lieber über Fisimatenten der Weiterbildung oder über TARMED streiten und weil uns schlicht die Ressourcen dazu fehlen. Dazu kommt, dass die Öffentlichkeit von Schlagwörtern geprägt ist. Es jagt ein Schlagwort das andere, die Medien propagieren schlecht recherchierte Informationen, und mit der Zeit fangen mehr und mehr Ärztinnen und Ärzte an, diesen Dingen zu glauben, ohne kritisch zu hinterfragen. Ein Beispiel hierfür ist die Salutogenese. Auf die Frage allerdings, was das genau sei, habe ich nie eine vernünftige Antwort bekommen. Aber diese Frage stellt niemand mehr. Wir müssen uns wieder bemühen, die ärztliche Uraufgabe klar und deutlich zu vermitteln.

Haben wir wegen Nebensächlichkeiten das Wesentliche aus den Augen verloren?

Wir haben, vermutlich seit den 80er Jahren, langsam aber sicher die Übersicht verloren, was überhaupt wichtig ist. TARMED und Weiterbildungsfragen sind Beispiele dafür. Immerhin ist es mir gelungen, dass an den Präsidentenkonferenzen wirkliche Aussprachen zwischen den politisch Verantwortlichen der FMH stattfinden können. Diese Umstellung ist auf eine grosse Zustimmung gestossen und deutet darauf hin, dass die politisch Verantwortlichen den FMH durchaus willens sind, sich mit grundsätzlichen Fragen auseinander zu setzen.

\section{Es gibt aber auch handfeste Interessen,} die in ganz verschiedene Richtungen gehen ...

Das ist richtig, aber letztlich sind es sehr wenige, die wirklich bis auf Biegen und Brechen ganz andere Wege gehen würden. Es kommt immer wieder ein Punkt, an welchem alle zusammenrücken und sagen, dass das Interesse und die Funktion der Gesamtärzteschaft erhalten werden müssen.

\section{Am lautesten sind trotzdem diejenigen, die auf Polarisierung hin drängen.}

Aber das ist immer so ...

... und sie haben eine Gefolgschaft, ...

... die überschätzt wird. Gerade in der Schweiz gibt es eine grosse Mehrheit der Schweigenden, die nichts sagen, wenn es gut geht.

\section{Die Urabstimmung wird vielleicht ganz andere} Landschaftsbilder der Ärzteschaft zeichnen, als dass uns gewisse suggerieren möchten?

Das kann durchaus sein. Ich vertrete in der Politik eine geradezu fundamentalistische Position und werde mich immer dem unterziehen, was die Mehrheit entscheidet, selbst wenn dies nicht zu meinem Vorteil wäre. In der Urabstimmung muss das Volk abstimmen, und wir werden dann sehen, wie die Landschaft wirklich aussieht. Es wird auch für unsere Mitglieder ein neues Gefühl sein, dass sie sich äussern 
können und dass ihre Stimme wahrgenommen wird. Ich habe Hunderte von Rückmeldungen erhalten, welche die Urabstimmung begrüssen, damit wir endlich wissen, was die Ärzteschaft wirklich will.

Im Grunde genommen gibt es unter den sogenannten Partner im Gesundheitswesen ausser der FMH nicht viele wirklich demokratisch funktionierende Organisationen.

Keine einzige. Eine Entscheidung über TARMED ist bei der sas die Sache einer Verwaltungsratssitzung. Auch bei H+ genügt im Prinzip die Entscheidung des Vorstands. Das ist in der FMH schlicht undenkbar, und das finde ich auch gut so. Wenn dieses Land etwas gewagt hat, und wenn es einen Grund gibt, dass es einen Sonderweg beschreitet, dann ist es diese Möglichkeit der direkten Intervention des Volkes, die kein anderer Staat in dieser Form kennt. Was dem Volk in der Schweiz recht sein soll, das muss auch für die Ärzte billig sein.

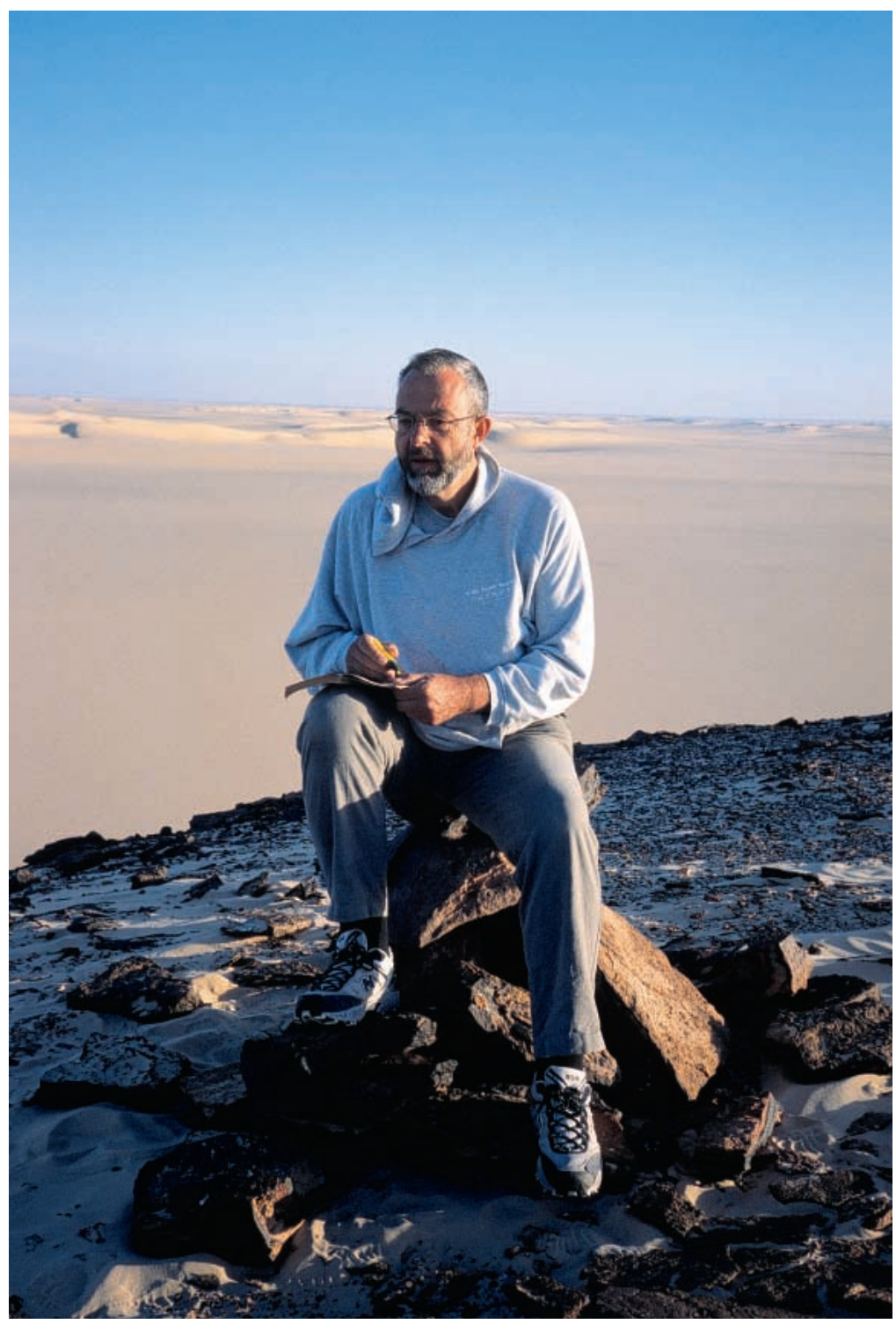

Dann sollten alle FMH-Mitglieder die Chance der Urabstimmung am Schopf packen.

Absolut, ich setze mich ein, den gesamten Prozess der Urabstimmung so zu gestalten, dass die Mitglieder der FMH wirklich Zeit haben, die Tarifstruktur zu studieren und dann ihren Entscheid zu treffen.

\section{Die FMH vertritt gleichzeitig die Interessen}

der Ärzte im Sinne einer Gewerkschaft und die Interessen der Medizin als einer übergeordneten Sache. Ist dieser Spagat schuld, dass die FMH manchmal eine weniger klare Haltung einnimmt, als wünschbar wäre?

Wenn wir Einkommen vertreten müssen, ist das ein schmerzhafter Spagat. Wenn die tarifarischen Kämpfe einmal durchgestanden sind und eine Rahmenordnung vorhanden ist, könnte es aber durchaus sein, dass andere Aspekte wieder zum Tragen kommen, die ein Zusammenleben dieser beiden Funktionen ermöglichen. Eine "Gewerkschaft» muss nicht immer nur Stundenlöhne vertreten, sie kann zum Beispiel auch die Qualität am Arbeitsplatz definieren, die vielleicht sehr viel wichtiger ist für die Arbeit selbst und für deren Resultat. Wir haben uns in den letzten zehn bis fünfzehn Jahren zuwenig um die Frage gekümmert, was wir unseren Mitgliedern anbieten müssen, damit sie ihr berufliches Umfeld verbessern können. Als Arzt muss ich in einem geordneten Umfeld arbeiten können, damit ich die Medizin interessant finde und auch Freude daran habe. Es ist in diesem Zusammenhang interessant festzustellen, dass die Rolle von Berufsorganisationen wie der FMH im Rahmen von Diskussionen zu Marktphilosophien neue Beachtung finden.

Wo sollten die nächsten Landmarken in unserem beruflichen Umfeld gesetzt werden? Bei den Arbeitszeiten oder beim Lohn?

Zunächst muss für einen Arzt eine gewisse Sicherheit existieren, wie sich sein Leben weiter entwickelt. Es kann sich niemand in einen beruflichen Werdegang wie z.B. Forschung investieren, wenn er nicht weiss, wie es in einem halben Jahr weitergeht. Ein Arzt muss wissen, dass er arbeiten kann, seine Familie muss das auch wissen. Vergessen wir nicht, dass diese Familien immer kritischer hinterfragen, sei es bei Männern oder bei Frauen. Was ich gerade bei jüngeren Ärztinnen und Ärzte feststelle, ist, dass die Sicherheit, den Beruf ausüben zu können, letzten Endes gegenüber der Einkommenshöhe immer wichtiger geworden ist. Viele Chefärzte müssten lernen, dass sie den meisten Gewinn - ich sage das ganz bewusst so - aus ihren Leuten ziehen, wenn sie ihnen eine Zukunftsperspektive und existentielle Sicherheit bieten können. Es kommen sekundäre Dinge hinzu wie die Tatsache, dass Ärzte gewöhnlich extrem negativ auf bürokratische Dinge reagieren, was sie ja auch ausweist und worüber Patienten froh sein können. Wenn die FMH in stetiger und beharrlicher Kleinarbeit diesen Kampf gegen eine unsinnige Bürokratie führen würde, würde sie wahrscheinlich grossen Applaus bei ihren Mitgliedern einholen. Es geht auch darum, die Mög- 
lichkeiten moderner Technologien auszunutzen und das, was heute mit Medical Informatics bezeichnet wird und Eingang in die Praxis und in den Spitälern findet, zu koordinieren, um damit einen kostendämpfend Effekt zu erreichen. Mit intelligenten Systemen kann z.B. auch Ärzten die Verantwortung überbunden werden, wie sie sich fortbilden sollen. Wir sollten uns hüten zu glauben, nur weil wir eine Fortbildungsordnung geschaffen hätten, seien nun die Probleme gelöst - weit davon entfernt: Bevor es nicht gelingt, Fortbildungsangebote zu schaffen, die Freude machen und als Notwendigkeit empfunden werden und die natürlich auch finanzierbar sind, ist nichts gewonnen.

Spielt bei diesen sicher sehr löblichen Bestrebungen nicht der zunehmende Einfluss der Industrie eine geradezu konträre Rolle? Auch die Industrie hat gemerkt, dass Fortbildung ein interessanter Markt ist, und wünscht sich, dass sich die Ärzteschaft gefälligst den im Markt gängigen Regeln zu fügen hat.

Das ist wahrscheinlich eine der gefährlichsten und bedenklichsten Entwicklungen in der medizinischen Wissenschaft, die nicht nur die Schweiz betrifft. Wenn diese Entwicklung so weiter geht wie bisher, kann sie das ganze System unterhöhlen und in sich zusammenbrechen lassen. Hier ist ganz klar der Staat aufgefordert, wieder zu begreifen, dass Forschung nicht etwas ist, aus dem man sich still und heimlich verabschieden und das man Sponsoren übergeben kann. Forschung war und ist auch heute noch ein wesentlicher Bestandteil des Service Public, d.h. Sache des Staats.

Im Vergleich zum Forschungsbudget einer grossen Firma wie Pfizer oder Novartis nimmt sich aber das Budget des Nationalfonds sehr bescheiden aus. Ich bin mir dieser Differenzen durchaus bewusst. Aber es gibt Leute in diesem Land, die begriffen haben, dass Forschung von Staates wegen gefördert und reguliert werden muss. Das schliesst Kooperationen mit der Industrie nicht aus, aber es darf nicht sein, dass die kommerziellen Interessen einer Firma massgeblich sind, was sie in einer medizinischen Fachdisziplin oder auf einem Fachgebiet geforscht wird. Das darf nicht passieren und hier kann wahrscheinlich nicht einmal ein Staat alleine das notwendige Gegengewicht setzen. Ohne dieses Gegengewicht wird aber Forschung innerhalb von zwanzig Jahren schlicht verkommen.

Liegt das Problem nicht noch tiefer, indem das Verständnis, was Wissenschaft eigentlich ist, verloren geht? Wenn gewisse Modeströmungen Scharlatanerie und Hokuspokus in der Medizin hochleben lassen, kommen Bestrebungen wie "Evidence-based Medicine", die das Wissenschaftliche wieder hervorzuheben versuchen, unter die Räder.

Seit mir jetzt jeder Pharmavertreter den Begriff "Evidence based Medicine» um die Ohren wirft, habe ich auch mit diesem Begriff etwas Mühe. Wir müssen da einige begriffliche Schärfungen vornehmen. Die allermeisten haben vermutlich nicht begriffen, dass die Medizin bis heute keinen methodischen Diskurs kennt, wie das zum Beispiel extrem für die Mathematik, auch für die moderne Physik und selbst für die Biologie gilt. Hier geht es nicht um wahr oder falsch, sondern darum, einen methodischen Diskurs zu entwickeln. Die Medizin hat diesen Diskurs nie in genügendem Ausmass geführt; wenn sie ihn führen würde, würde sich zeigen, dass es in diesem und jenem Bereich tatsächlich Hokuspokus gibt. Ich würde so weit gehen zu behaupten, dass wahrscheinlich Medizin in gewissen Aspekten immer schon Hokuspokus gewesen ist und dass das vielleicht sogar so sein muss. Aber wenn dieser Diskurs sauber geführt würde, würden wir wissen, wo es den Hokuspokus braucht. In der jetzigen, völlig diffusen und begrifflich unscharfen Situation ist die Gefahr, dass am Ende alles Hokuspokus wird. Ein Diskurs ist ein nach gewissen Regeln stattfindender gegenseitiger Austausch. Wir deutsch Sprechenden haben grosse Mühe mit dem Begriff des Diskurses, im Gegensatz zu französisch Sprechenden, die sehr viel präziser wissen, was "un discours" ist. Uns geht diese begriffliche Schärfe leider ab, und das ist ein Defizit, das ich verspüre, seit ich Medizin mache. Es mag vielleicht erstaunen, aber ich wollte ursprünglich Mathematik studieren. Aus verschiedenen Umständen, die hier keine Rolle spielen, ist es nicht dazu gekommen, aber Mathematik ist für mich immer noch diese stille ferne Geliebte geblieben. Das vielleicht als Erklärung, warum ich mir wünsche, dass wir unsere Diskussionen auf dem Niveau und mit der semantischen Klarheit halten würden, wie das Mathematiker tun; wir könnten uns da ein grosses Stück abschneiden. Offen gestanden, ich kann mir nicht vorstellen, dass eine Versammlung der Vereinigten Schweizer Mathematiker sich so abspielen würde, wie unsere Ärztekammern. Ich denke gerne an das Votum eines jungen VSAO-Delegierten zurück, der in einer jener hitzigen Auseinandersetzungen plötzlich das Votum abgegeben hat: «Also, meine Damen und Herren, hier geht's doch erst einmal darum, dass wir Datenbanken haben. Sind nun diese Datenbanken richtig programmiert worden, sind die Kalkulationen richtig?» Der hat mir gefallen. Das ist die Art von Diskurs, wie ich ihn mir überall wünsche.

\section{Müsste dieser Diskurs schon im Studium integriert werden?}

Das könnte man, es würde aber eine grundsätzliche Umstellung des Studiums bedeuten. Alle Studiengänge zeigen eine Tendenz zur Überbelastung und Verschulung. Das Medizinstudium heute, wie ich das bei zweien meiner Kinder gesehen habe, ist in einem erschütternden Ausmass verschult. Wir müssten all diese starren Strukturen aufbrechen und die im ursprünglichen Sinne des Wortes zu verstehende akademische Musse schaffen, damit jüngere Leute wieder Freude am lernen finden und einen Diskurs, an welchem sie eigentlich sehr interessiert sind, führen können. 
Um einen Diskurs zu führen, müssten die Studenten auch einen Gesprächspartner haben, ...

... den sie im Dozentenkollegium nicht finden. Hinzu kommt, dass eine akademische Ausbildung nicht möglich ist, wenn alle Jahre auf Prüfungen hin eine Unmenge von Daten abspeichert und zum geeigneten Zeitpunkt in möglichst konfektionierter Form reproduziert werden muss. Man muss sich eingestehen, dass heute das Medizinstudium über weite Strecken bestenfalls einer Fachhochschule aber sicher nicht mehr einem universitären akademischen Denken gerecht wird, wie es vor hundert Jahren noch eine gewisse Selbstverständlichkeit war.

Hätten wir auch etwas von den Rechtswissenschaften zu lernen?

Wir könnten davon sehr viel lernen, z.B. im Umgang mit Begriffen und in der Einordnung in ein System. Es ist immer wieder faszinierend, Juristen zuzusehen, wie sie einen Sachverhalt zuerst semantisch, dann topologisch und strukturell gliedern. Wenn sie das bewusst und nicht nur maschinell tun, sind Juristen hochinteressante Gesprächspartner.

Schade, dass man bei Ärzten vielfach einen AntiJuristen-Reflex feststellt ...

Ach, den habe ich überhaupt nicht ...

Wir Ärzte sind Weltmeister in der Arzt-PatientenBeziehung. Aber sobald wir diese Beziehung verlassen und in ein soziales Umfeld treten, sind wir tollpatschig. Wie könnten wir uns im Sozialen etwas geschickter bewegen?

Ärzte haben sich eigentlich schon früher sozial engagiert und tun dies auch heute noch. Das wird vielleicht nicht so wahrgenommen und auch weniger gewürdigt. Ein Allgemeinpraktiker in einer ländlichen Region steht gewöhnlich als Feuerwehrarzt zur Verfügung und ist am grossen Sängerfest anwesend. Ärzte übernehmen schon noch soziale Aufgaben und werden für das auch in der Bevölkerung estimiert. In Urbangebieten hingegen ist diese herkömmliche Form von sozialem Engagement nicht mehr möglich. Hier müssen Ärzte lernen, sich in sozialen Gebieten zu engagieren, zum Beispiel in Patienten-Interessengruppen.

Kehren wir zum eigentlichen Medizinischen zurück. Sie haben vorhin die Fortbildungsordnung in ihrer Bedeutung, und ich glaube zu Recht, etwas relativiert. Müsste man nicht auch das grosse andere Schlagwort, die Qualitätssicherung, als Seifenblase entlarven?

Sicher, eine Seifenblase ist ein dünnes Gebilde mit Luft drin ... das ist ein Aspekt. Was die Ärzteschaft aber zuwenig begriffen hat ist, dass die gängige Form der Qualitätssicherung, die sich des Anekdotischepisodenhaften bedient, heute nicht mehr genügt. Dort, wo ein Arzt noch die gesamte Verantwortung trägt, typischerweise ein Chirurg an einem mittleren Spital, ist die Qualitätssicherung einigermassen ein- fach. Heute haben wir aber vielfach eine völlig andere Situation, wo die Qualität der medizinischen Leistung nicht nur durch den Arzt, sondern von vielen anderen Berufen mitdefiniert wird, und wo der Arzt eine ganz andere Rolle spielt, als das konventionell der Fall gewesen ist.

\section{Aber es ist immer noch so, dass der Chirurg} operiert, Hilfsberufe hin oder her. So sehr hat sich alles auch wieder nicht geändert.

Nun gut, ohne den Anästhesisten kommt der Chirurg wahrscheinlich nicht mehr so gut aus, gerade wenn es darum geht, den kritisch kranken Patienten am Leben zu erhalten. Heute findet die Verrichtung der ärztlichen, chirurgischen Leistung auf dem Hintergrund einer Zusammenarbeit mit anderen Disziplinen statt, welche die Voraussetzungen für ein sicheres Umfeld garantieren. Das ist etwas Neues. Ich möchte nicht so verstanden werden, dass die gesamte Verantwortung jetzt von den Ärzten, Chirurgen weggegangen sei, aber die Funktion des Arztes ist eine andere geworden. Es ist nicht mehr eine Sache nur zwischen dem Patienten und dem Arzt, sondern zwischen dem Patienten und einem Arzt als Hauptverantwortlichen und vielen anderen Fachleuten. Diese Verschiebung ruft zwangsläufig nach mehr Transparenz. Wenn das letzte Jahrhundert eine grosse Tendenz hatte, dann war es die, alle Vorgänge, alle Entscheidungen allen sichtbar zu machen. Diese für die grossen Demokratien unerlässliche Transparenz ist von den Ärzten als Bedrohung oder als Zumutung wahrgenommen worden. Sie haben nicht erkannt, dass auch eine grosse Chancen darin liegen kann, das Ganze auf den Kopf zu stellen.

Mit der Transparenz ist der Aufbau eines ganzen

Erkenntnis- oder Kontrollapparates notwendig.

Diese Frage bezieht sich auf ein bestimmtes Modell der Qualitätssicherung oder der Qualitätskontrolle, das darin besteht, mit technischen Instrumenten Menschen von morgens früh bis abends spät zu beobachten und die Fehler mit einer Strichliste zu erfassen. Eine Qualitätsverantwortung, die auf solch einem System aufbaut, wird mit Sicherheit nicht zum Tragen kommen, darauf müssen wir gar nicht eingehen.

Was schwebt Ihnen denn vor?

Mir schwebt ein System vor, wo ich gezwungen bin, mich selber den Dingen, die ich tue, zu stellen und sie nicht mehr zu verdrängen. Zum Beispiel muss ich als Chef einer Chirurgischen Klinik sehen, dass bei mir von X Patienten mit einer bestimmten Situation Y eine Komplikation haben oder sterben. Wenn ein Doktor sich auf dem Hintergrund von anderen Kollegen sehen kann, ist das eines der wirkungsvollsten Instrumente. Das geht nur im stillen Kämmerlein, wenn der Arzt mit sich selber allein ist. Falls eine Fehlerkultur entsteht, wäre der nächste Schritt, dass auch ein Team Fehler erkennen und diskutieren kann und daraus lernt. Mit einer Schuldzuweisungskultur ist Qualitätssicherung zum Scheitern verurteilt. 
Wo stehen wir konkret? Es war einmal die Rede von einer nationalen Datenbank, die Critical Incidents repertorieren soll. Es wurde aber dann der juristische Einwand vorgebracht, dass eine solche Datenbank vor dem Untersuchungsrichter nicht geschützt werden könne.

Eine solche Datenbank kann sinnvollerweise nur dann geführt werden, wenn sie keine personifizierbaren Daten enthält. Auf eidgenössischer Ebene ist es auch belanglos, die Namen zu kennen, es interessiert nur, welche Fehler unter welchen Umständen passieren.

Damit findet aber kein Feedback statt.

Richtig, mit diesem System muss sich jeder selber einordnen. Wenn natürlich der unerwartet grosse Wunsch nach personifizierten Daten vorgebracht würde, wovor ich allerdings den grössten Respekt hätte, dann gäbe es technische Lösungen dafür. Für mich liegt der Schwerpunkt aber im Moment darin, die Realität abzubilden.

In diesem Gespräch geben Sie ein anderes Bild von sich, als man vielleicht vom TARMED-Epos her gewöhnt ist. Es wäre zu hoffen, dass dieses Bild auch gute Leute motivieren könnte, Berufs- und Gesundheitspolitik zu betreiben. Könnten noch andere Anreize geschaffen werden, um gute Leute für solche Tätigkeitsfelder zu gewinnen?

Wer in die Standespolitik hineingehen möchte, sollte verschiedene Voraussetzungen mitbringen. Erstens braucht es Interesse und Verständnis für politische Prozesse. Wer mit einer fundamentalistischen Haltung kommt, wird Misserfolge erleben. Dann braucht es doch eine gewisse Erfahrung in der Medizin. Es kann keiner nach einem Jahr Klinik in den Zentral- vorstand Einsitz nehmen, so wünschenswert es an sich ist, wenn junge Leute kommen. Aber es braucht ein gewisse Vertrautheit mit dem medizinischen Alltag. Wer Politik macht, ist potentiell massivster Kritik ausgesetzt, und dies zu einem Stundenansatz, der nicht primär motivierend ist. Wenn die Beanspruchung durch die standespolitische Tätigkeit ein gewisses Mass überschreitet, und das ist heute viel schneller der Fall als früher, dann stellt sie die medizinische Tätigkeit in Frage, sei es die Führung einer Praxis, sei es eine Chefarztfunktion, und dann kommen die Fragen der Abfindung und die der beruflichen Perspektiven. Wer sich in den Zentralvorstand wählen lässt, muss seine Berufstätigkeit reduzieren und riskiert bei einer Abwahl, mit leeren Händen und am Ende einer Laufbahn dazustehen. Das wird viele Leute zurückhalten, sich für eine Berufsorganisation wie die FMH zu engagieren. Wenn nicht mehr Mittel eingesetzt werden, ist das Problem nicht lösbar. Anderseits darf es nicht passieren, dass im Leitungsgremium der FMH nur Leute sitzen, die irgendeinmal Patienten behandelt haben, aber sonst nur noch am Schreibtisch tätig sind. Um die Medizin vertreten zu können, muss man die Luft von Spital oder Praxis kennen; die wechselt praktisch von Woche zu Woche, und wer dies nicht wahrnehmen kann, wird sehr schnell an der Realität vorbei politisieren. Die Kunst wird sein, eine Situation zu schaffen, die genügend Zeit für die berufliche wie für die berufspolitische Tätigkeit zulässt. Irgendeinmal wird sich die Ärztekammer aber klar werden müssen, dass es mit den Hauptverantwortlichen und ihrer existentiellen Lage nicht so weiter gehen kann, wie bisher.

Besten Dank für dieses Gespräch!

Interview: Markus Trutmann 